\title{
Influence of force and torque feedback on operator performance in a VR-based suturing task
}

\author{
L. Santos-Carreras ${ }^{\mathrm{a} *}$, R. Beira ${ }^{\mathrm{a}}$, A. Sengül ${ }^{\mathrm{a}}$, R. Gassert ${ }^{\mathrm{b}}$ and H. Bleuler ${ }^{\mathrm{a}}$ \\ ${ }^{a}$ Laboratoire de Systèmes Robotiques École Polytechnique Fédérale de Lausanne (EPFL), Lausanne, Switzerland; ${ }^{b}$ Rehabilitation \\ Engineering Lab ETH Zürich, Switzerland
}

(Received 1 February 2010; final version received 18 June 2010)

\begin{abstract}
The introduction of Minimally Invasive Surgery (MIS) has revolutionised surgical care, considerably improving the quality of many surgical procedures. Technological advances, particularly in robotic surgery systems, have reduced the complexity of such an approach, paving the way for even less invasive surgical trends. However, the fact that haptic feedback has been progressively lost through this transition is an issue that to date has not been solved. Whereas traditional open surgery provides full haptic feedback, the introduction of MIS has eliminated the possibility of direct palpation and tactile exploration. Nevertheless, these procedures still provide a certain amount of force feedback through the rigid laparoscopic tool. Many of the current telemanipulated robotic surgical systems in return do not provide full haptic feedback, which to a certain extent can be explained by the requirement of force sensors integrated into the tools of the slave robot and actuators in the surgeon's master console. In view of the increased complexity and cost, the benefit of haptic feedback is open to dispute. Nevertheless, studies have shown the importance of haptic feedback, especially when visual feedback is unreliable or absent. In order to explore the importance of haptic feedback for the surgeon's master console of a novel teleoperated robotic surgical system, we have identified a typical surgical task where performance could potentially be improved by haptic feedback, and investigate performance with and without this feedback. Two rounds of experiments are performed with 10 subjects, six of them with a medical background. Results show that feedback conditions, including force feedback, significantly improve task performance independently of the operator's suturing experience. There is, however, no further significant improvement when torque feedback is added. Consequently, it is deduced that force feedback in translations improves subject's dexterity, while torque feedback might not further benefit such a task.
\end{abstract}

Keywords: haptic IO; force feedback; torque feedback; teleoperated surgery; psycophysics; suture accuracy

\section{Introduction}

Minimally Invasive Surgery (MIS) procedures are applied for the same purposes as open surgery procedures, but with the advantage of being less invasive. They usually utilise endoscopic tools (long and narrow instruments) inserted through small incisions into the patient's body. Some benefits of MIS are reduced pain, trauma, risks of infection and haemorrhage, as well as a reduced hospital stay, thus significantly reducing overall costs (Mack 2001) and complications. Despite these benefits, MIS is very demanding for surgeons, as

(1) the field of view and workspace are limited;

(2) there is only $2 \mathrm{D}$ vision (depth perception is lost);

(3) direct hand contact is lost;

(4) manipulation of the laparoscopic tools under modified hand-eye coordination must be trained.

Some of these drawbacks can be overcome with the introduction of telemanipulated robotic surgical systems, composed of the following two main elements: (1) the surgeon console acting as a master, and (2) the patientside surgical robot acting as a slave. Surgeons can see 3D images of body organs, thanks to an endoscope with two integrated cameras and a stereo projector integrated in the surgeon's master console. Moreover, the robotic instruments are easily manipulable (Guthart and Salisbury 2000). Furthermore, these robotic systems offer new possibilities, such as tremor filtering and intelligent-assistance functions like virtual guidance, monitoring of the manipulation forces etc. Robotic surgery has been increasingly performed in the last few years, especially for bariatric (Jacobsen et al. 2003; Moser and Horgan 2004), urologic (Dasgupta et al. 2005) and cardiothoracic surgery (Bodner et al. 2004). Despite all these advantages, if the properties of the distal organs are not properly rendered to the surgeon, his/her performance can be severely degraded. The term transparency is used to quantify the fidelity with which the visual and haptic properties of the distal objects are rendered to the surgeon (Lawrence et al. 1996). In the case of force

*Corresponding author. Email: laura.santoscarreras@epfl.ch 
feedback, an ideal transparent system would render force and position information as if the master handle was directly in contact with the slave manipulator. In other words, the physical properties of the teleoperation system itself, such as inertia, compliance, friction, time delays and other artifacts, should be 'invisible' to the user. This leads to the definition of transparency as a transfer function. Nowadays, most robotic surgical systems do not provide suitable haptic feedback. Therefore, their transparency is considerably reduced and the forces applied to the tissue are still heavily reliant on visual cues.

The main constraint for including force feedback in a robotic surgical system is the increase of complexity and cost. The surgeon's master console requires an actuator and a position sensor per active degree of freedom (DOF). In addition, the slave robot must contain force sensors to measure interaction forces between surgical tools and tissues, and possibly also tactile sensors to render surface properties. A simple tool-organ interaction, such as exploring the surface of an organ, involves at least three forces and torques each. Furthermore, most of surgical tasks would require an extra (seventh) DOF to open and close the surgical tool. Taking into account this, and that surgeons generally use both hands, the surgeon's master console should then provide 14 active DOF of force feedback. In other words, the system should include 14 actuators, 14 position sensors in the surgeon's master console and in the slave robot tools to measure a total of 14 DOF of force, which would considerably raise system complexity and cost, thus making it difficult to control and unaffordable for the majority of hospitals.

To design the optimal haptic device for a surgeon's master console in a robotic surgical system, the catalogue of solutions should be task-specific. Therefore, the surgeon's actions must be decomposed into basic hand gestures in order to identify the active DOF crucial for the surgeon and the DOF that can be inactive to decrease the overall complexity and cost. The most frequent tasks executed by surgeons during a common surgical procedure are resection, grasping, suturing, ablation and injection (Nio et al. 2002). Most of these tasks are carried out by translating the end effector in three dimensions. Rotations are seldomly used except for orienting the surgical tool that remains almost invariable during the translations. This implies that the surgical device must have seven DOF for positioning tools (three DOF for translations, three for orientation and one for the grip movement). However, it might be necessary to provide force feedback only in the translational DOF and in the grip movement, but no torque feedback because the orientation is rarely changed as the task is performed. Nevertheless, while suturing, to insert the curved needle into the tissue, surgeons execute a pure rotation of their wrist around its pronosupination axis (Figure 1) (Seki 1988; Iwamoto et al. 1993). Therefore, for this specific task, torque feedback could help the surgeon to correct his trajectory and to apply the correct forces, thus improving suture quality.

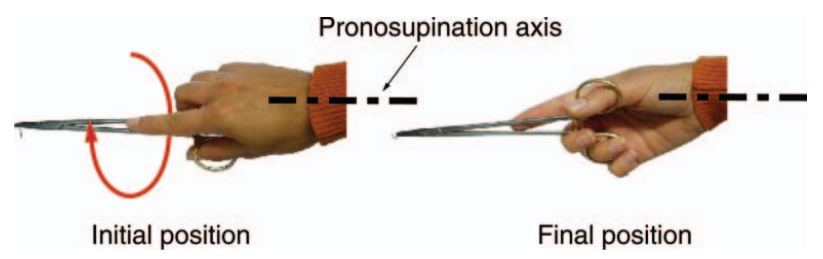

Figure 1. Wrist rotation around the axis of the forearm during needle insertion (pronosupination).

The first commercially available haptic devices provided only three active DOF of force feedback in the translation axes ( $x, y$ and $z$ ) (Massie and Salisbury 1994; Grange et al., 2001). This kind of device can be used in many applications in which tool orientation does not have to be changed and users only need to move in the $3 \mathrm{D}$ space, or where it is sufficient to record and display orientations.

The well-known companies are adding three DOF of torque feedback as well as providing kinesthetic feedback in six DOF (Hayward et al. 1998; Cohen and Chen 1999). The addition of these extra three DOF implies the integration of three more actuators and sensors that considerably increases both inertia and controller complexity and frequently results in a loss of transparency (Barbagli and Salisbury 2003). In order to reduce complexity while keeping the full number of DOF (six or seven per hand), force feedback can be realised only in a subset of the DOF, usually for the translational forces. The remaining DOF are only equipped with position sensors.

Examples of commercial devices presenting this actuator/sensor asymmetry are the PHANToM Omni (Sensable Technologies, Woburn, MA, USA) with six sensors and three actuators and the omega.7 (Force Dimension, Nyon, Switzerland) with seven sensors and four actuators. These devices are suitable for many applications, including robotic surgical platforms, and their cost is considerably lower than the fully actuated versions (Seibold et al. 2008).

In their pioneering work, Barbagli and Salisbury (2003) considered the effect of sensor/actuator asymmetries in haptic devices. Their results reveal that sensor/actuator asymmetry may lead to non-conservative forces in specific situations, reducing the realism of Virtual Reality (VR)-based devices. Semere et al. (2004) performed an experimental study to determine the effect of this sensor/actuator asymmetry during a blunt dissection. One of the conclusions to be drawn from the former work is the improvement of the performance after adding partial force feedback. In a later study (Verner and Okamura 2007), performance and applied force during a peg-in-hole task were analysed to evaluate the effect of the grip-force. In this case, feedback of the grip-force helped subjects to apply the minimum force required to hold the peg. Regarding the addition of torque feedback, the results of recent studies differ depending on the application. Verner and Okamura (2009) report that 
performance with force feedback alone is close to the one with force and torque feedback for simple tasks such as drawing in a 3D virtual environment. However, in other applications in which forces and torques are decoupled, the torque information might be crucial. For instance, it is proven that the torque feedback provided by the steering wheel of the car has a direct implication on driver's performance because it carries information related to the vehicle dynamics (Toffin et al. 2007). Furthermore, if the visual information is limited or unreliable, users mainly rely on the haptic information (Ernst and Banks 2002). For example, during screw insertion in a teleoperated spinal-fusion surgery, both torque and force feedback are necessary to successfully achieve the task (Lee et al. 2009). The results of these studies show that the effect of actuator/sensor asymmetry mainly depends on the task to be carried out. The effect of torque feedback in the pronosupination DOF of the wrist on suture performance has not been investigated and can only be determined by performing experiments involving this specific task.

The goal of the work presented here is to determine if torque feedback should be added into the surgeon's haptic console of a teleoperated robotic system for endoluminal surgery. The results will be used to determine the design specifications of both the haptic device and the slave robot force sensor for a telemanipulated robotic surgical system.

This paper is structured as follows. First, in Section 2.1 , we describe the haptic device and the torque feedback module developed for the experiment. In Section 2.2 we describe the virtual environment developed for the test. In Section 2.3 we present how suture performance is measured in a pinpoint suture task. In Section 2.4 we provide details about the followed experimental protocol. Experiment results are presented in Section 3. Discussion and conclusions close the paper.

\section{Methods}

The major goal of this work is to investigate the influence of force and torque feedback in a tele-robotic surgery system using a virtual reality testbed. The choice of a VR testbed was motivated by the desire for well-controlled and repeatable conditions that can be recreated. This solution permits preservation of the same conditions for all subjects running the experiment (e.g. tissue properties and tool orientation). In addition, the VR-based testbed gives the possibility to randomly mix feedbacks during the experiment and consequently subjects cannot be aware of the next feedback that will be provided.

The results might be affected by the slave system limitations (e.g. noisy force measurement, bandwidth or sensor resolution) if an artificial tissue material and a tele-operated robot are used. As the proposed experiment focuses only on how force and torque feedback affect subject performance, a virtual reality testbed was found to be the optimal solution.

\subsection{Haptic device and torque feedback module}

The haptic device used in the experiment was an omega.3. However, for this specific task, at least four DOF are required: three translations to position the suturing needle on the suture location and an extra DOF to rotate the arc-shaped needle around its centre. For this reason, a specific handle allowing this extra DOF was developed (Figure 2(a)). According to the wrist mobility anthropometric data, at least $220^{\circ}$ should be provided to allow almost a full rotation around the pronosupination DOF of the wrist (Pheasant 1986). In addition, a conventional needle holder was used in the construction of the handle to preserve the ergonomics of a normal suture.

To guarantee a similar performance of the haptic device for all the suture locations, the suture points were located within the boundaries shown in Figure 2.

The forces measured during needle insertion and soft tissue indentation reported in Okamura et al. (2004) and Samur et al. (2007) were taken into account to select the actuator. An RE25 motor (Maxon Motor AG, Sachseln. Switzerland) with a magnetic encoder (1000 CPT) was chosen. The needle used in the simulation is a conventional arc-shaped needle of $14.5-\mathrm{mm}$ diameter as used in Iwamoto et al. (1993). In the investigation reported by Okamura et al. (2004) the maximum force measured was 2.3 $\mathrm{N}$ on the needle tip. Taking into account that the needle diameter is $14.5 \mathrm{~mm}$, the maximum peak torque that the motor should provide is $30 \mathrm{mNm}$, which is very close to the nominal motor torque of $29.2 \mathrm{mNm}$.

The torque feedback module was carefully designed to minimise the effects on device transparency. By definition the transparency transfer function of the haptic device is the ratio of the transmitted impedance to the simulated impedance (McJunkin et al. 2005). Therefore, the kinematics and the dynamics of the haptic device are part of the transparency transfer function. This transfer function should ideally be an identity matrix; however, this requires a perfect compensation of friction losses, inertia and gravity. The omega. 3 provides an accurate gravity and inertia compensation that is maintained in translation space by coupling passive and actuated components together. In order to preserve the performance of the entire device, the mass of the torque feedback module was adjusted in the compensation algorithms. In addition, the torque module was individually calibrated to ensure repeatable and optimal precision and performance. Furthermore, the chosen RE 25-DC motor and transmission components present very low friction, inertia and backslash (Table 1).

An electronic interface was developed in order to avoid an additional Universal Serial Bus (USB) connection. This solution permits the use of the original motor amplifiers of the haptic device (force dimension), and consequently a perfect synchronisation with the rest of the actuators can be guaranteed. 

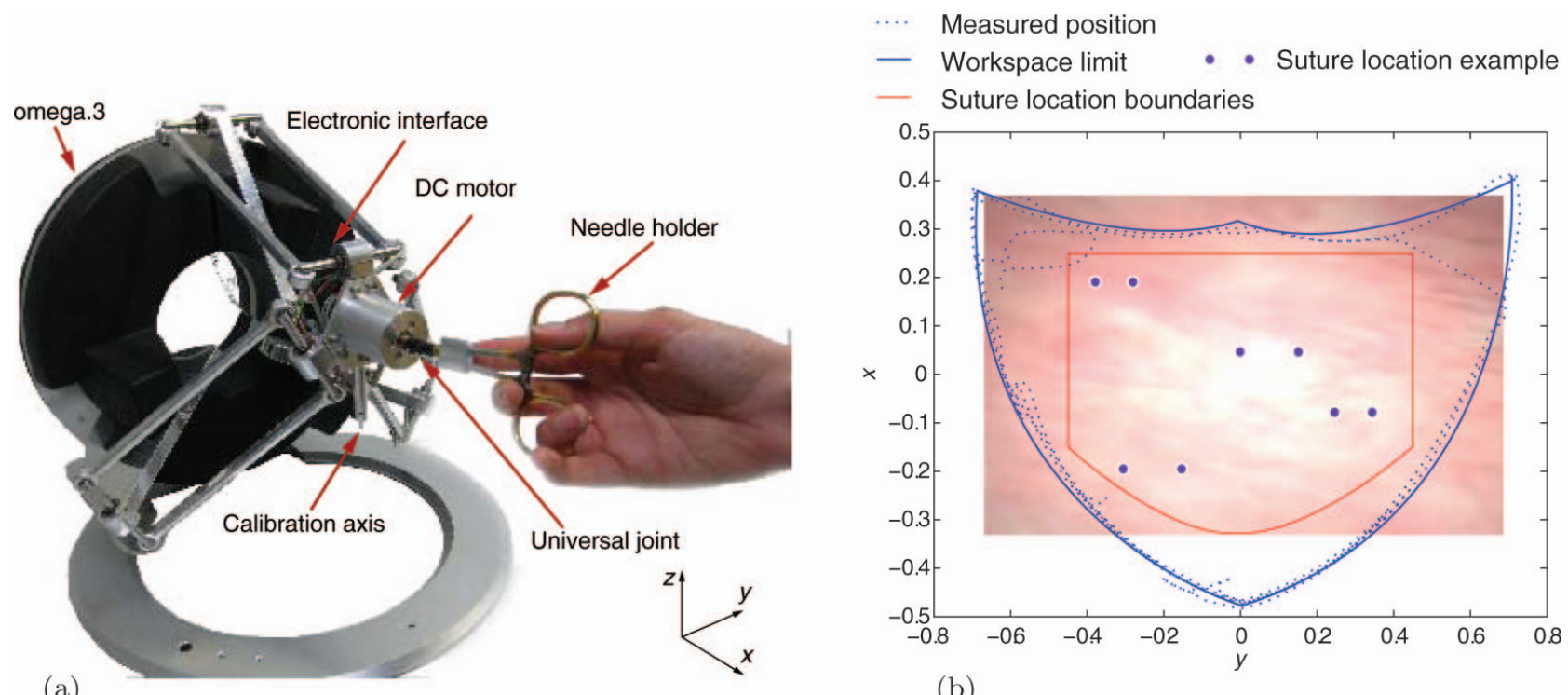

(b)

Figure 2. Haptic device used in the experiment and task workspace. (a) The customised handle added to the omega.3, provides torque feedback in the pronosupination DOF of the wrist. (b) Suture location boundaries with respect to the omega. 3 workspace in the horizontal plane passing by its centre $(z=0)$.

As the aim of this experiment is to determine whether the specific torque along the pronosupination DOF improves performance, the other two rotations for the wrist orientation are kept invariant in the simulation. Therefore, they are not tracked by any position sensor. To avoid a transparency decrease due to the constrained rotations, the suture points are always parallel to the base of the omega.3, as can be seen in Figure 2. Therefore, there is no need to change the orientation when performing this specific suture task.

The preliminary trials showed that a rigid horizontal tool resulted in an unnatural wrist posture. For this reason, a universal joint was added between the actuator and the needle holder to allow for small deviations in the subject wrist orientation (Figure 2(a)). Nevertheless, subjects were instructed to keep the orientation of the other rotation axis essentially unchanged. Alignment deviations of a few angular degrees were tolerable in the framework of these experiments.

Table 1. Characteristics of the torque feedback module components.

\begin{tabular}{lccc}
\hline Parameters & Motor & Universal joint & Handle \\
\hline Radial play $(\mathrm{mm})$ & 0.025 & Backlash free & Backlash free \\
Axial play $(\mathrm{mm})$ & 0.05 & Backlash free & Backlash free \\
Weight $(\mathrm{g})$ & 130 & 0.7 & 23 \\
Inertia $\left(\mathrm{gcm}^{2}\right)$ & 10.5 (rotor) & 0.03 & - \\
\hline
\end{tabular}

\section{2. $\quad$ VR testbed}

The VR testbed was kept as simple as possible to reduce the influence of unknown factors. In the experiment, sutures are performed with only one hand. Although surgeons usually grip the tissue with one hand and suture with the other, only one hand is used during the test to avoid the gripping having an impact on the results. For this reason, the virtual tissue was flat. This factor differs from a real suture task where surgeons insert the needle into the edge of the gripped tissue.

The VR testbed consists of a soft tissue and a needle holder with a fixed, curved needle (Figure 3 ). The needle holder is a virtual copy of the real holder used in the haptic device. The surgical needle is an arc-shaped needle, commonly used for skin, peritoneum and muscle sutures. The shadow of the tool on the tissue was added to provide additional visual perception of depth to the user. It was found to be a crucial cue for users to target the given entrance point of the suture during the experiment.

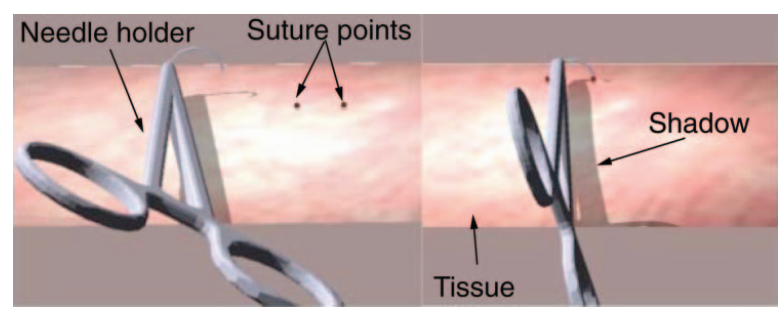

Figure 3. Virtual suturing testbed. Users used the shadow to position the needle near the indicated entrance point. The suture was performed towards the indicated exit point. 


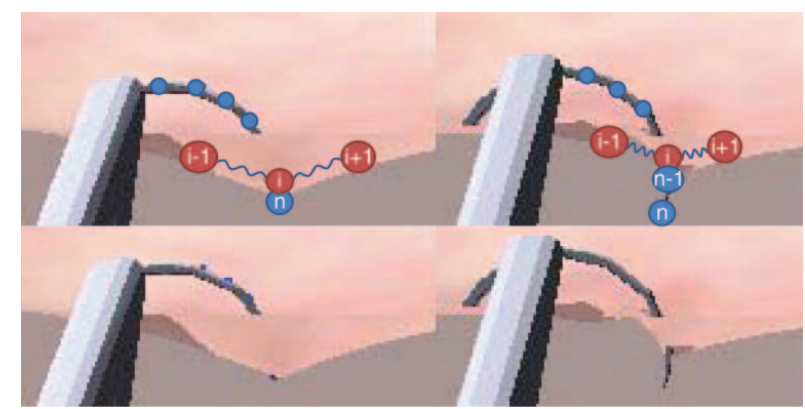

Figure 4. Simulation of the needle insertion, where $n$ represents the needle nodes and $i$ represents the tissue elements.

The soft tissue was modelled as a mass-spring-damper mesh and the needle arc was decomposed into 10 connected segments. As the haptic device represents the needle holder, position and rotation are read from the device and the position of each needle node is calculated using a trigonometric transformation. A bounding sphere is created around the needle tip using the length of the spring of the soft tissue. Whenever a tissue node is located inside this bounding sphere, the neighbour masses become collision candidates.

The collision detection has two different modes depending on the needle orientation. The first collision mode simulates the interaction of the concave part of the needle with the tissue when needle insertion cannot be performed. The second collision mode simulates the needle insertion. This is a complicated procedure to simulate with several states depending on the number of needle nodes inserted and the needle trajectory (e.g. penetrating or exiting the needle at the entrance point). We used a similar procedure, such as the one proposed by Wang et al. (2008), to simulate the insertion of the needle in a vessel. As shown in Figure 4, the pierced mass of the tissue element $i$ will initially follow the closest needle node $n$. When the length of the adjacent springs overcome a threshold, the tissue element $i$ will follow the next needle node $n-1$.

The haptic device is impedance-controlled. As can be seen in Figure 5, user movements are measured and thereafter the interaction with the virtual tissue for that specific needle movement is calculated. The torques that might be generated by the device motors are then transmitted to the omega control board. With this approach, users feel the forces from the dynamics and statics of the simulated environment in response to moving the device (Hayward and MacLean 2007). The simulation was run on a Mac Book 5.1 laptop with an Intel Core 2 Duo $2.4 \mathrm{GHz}$ CPU. The laptop graphic card was a NVIDIA GeForce 9400 M 256 M GPU. The haptic and graphic loops were updated at $1 \mathrm{kHz}$ and $30 \mathrm{~Hz}$, respectively.

\subsubsection{Insertion model}

The task carried out in the experiment is a simple pinpoint task of passing the needle through two points. Between the input and output points the needle is hidden by the tissue. The only information that users have is their self-perception of the movement and the haptic feedback (if given). As the experiment is performed to investigate whether the torque feedback might help in this specific surgical gesture, the force model of the tissue follows the same correlation of forces of a real needle insertion. However, interactions between needles and living tissues are very complex:

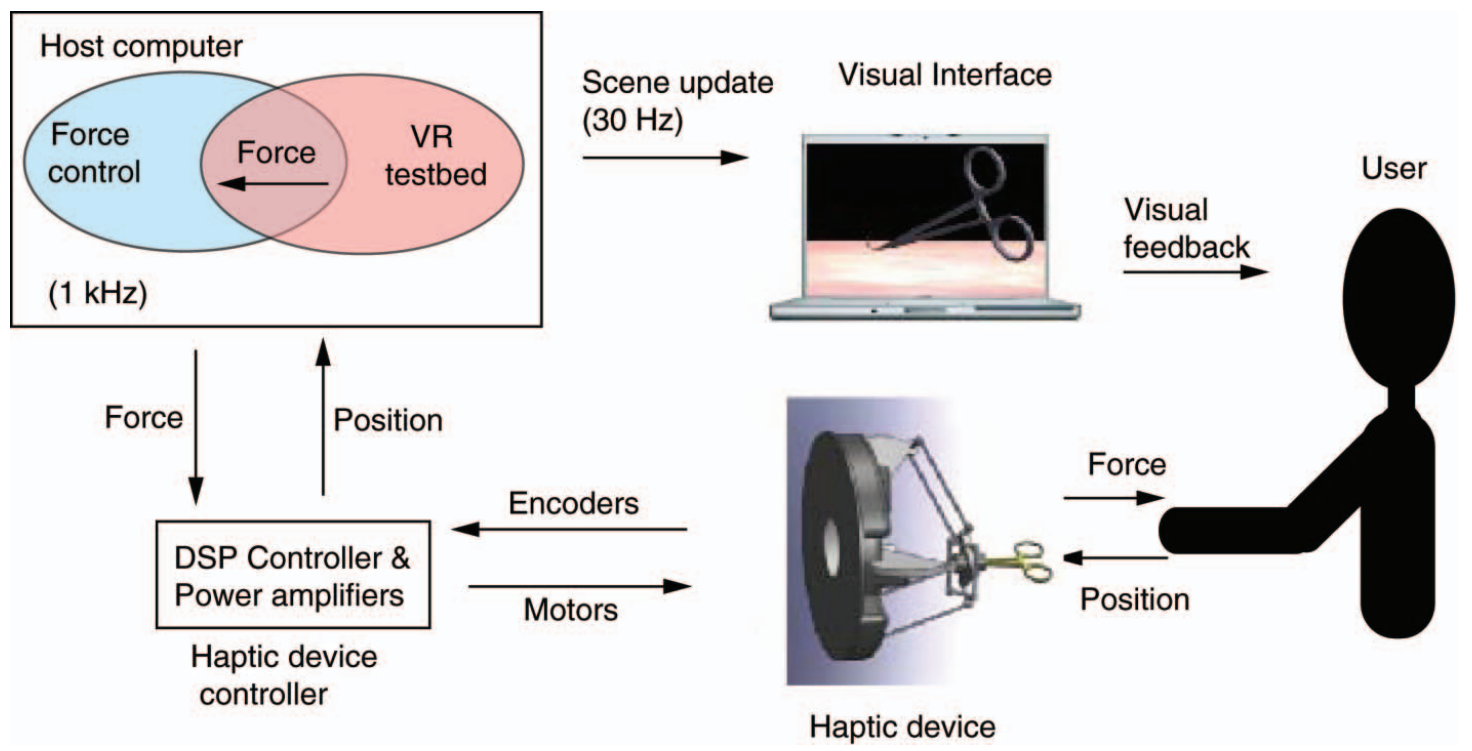

Figure 5. Control scheme of the overall experimental setup. 


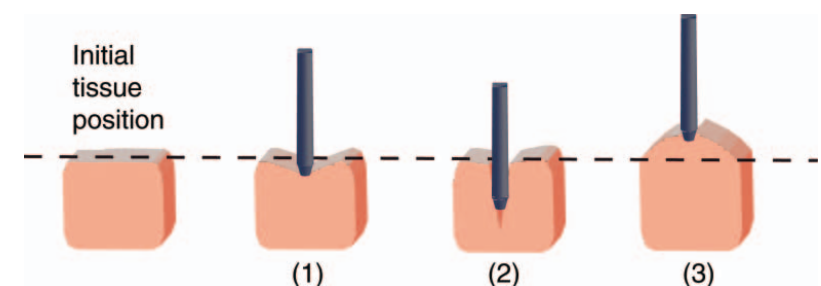

Figure 6. 1 DOF needle insertion phases described in Okamura et al. (2004), Barbé et al. (2007a) and Barbé et al. (2007b).

(1) The different layers of the tissue crossed by the needle are inhomogeneous and so the mechanical properties cannot be described by an elastic isotropic model.

(2) Tissues are organ-dependent.

(3) The insertion forces change with the needle shape.

This inherent complexity in living tissues has been simplified in the literature. Most of the current needle insertion models are based on measurements of straight needle insertions that move in only one DOF (Simone and Okamura 2002; Okamura et al. 2004; Abolhassani et al. 2007; Barbé et al. 2007a, 2007b). In these experiments it was found that a needle insertion follows at least three phases. These phases are represented in Figure 6, and described as follows:

(1) Contact and deformation.

(2) Once the energetic threshold is overcome, the needle perforates the surface. While the needle is inserted, the damping forces attract the tissue along the needle shaft.

(3) The needle is extracted. Again the tissue adheres to the needle shaft and follows its movement.

For this experiment the insertion of the needle follows the same phases. Forces rendered to the user are calculated similarly to the model described in Okamura et al. (2004) and Barbé et al. (2007a). In latter studies the needle insertion force model is a combination of stiffness, damping and puncture forces that takes into account the needle depth in the tissue. However, in both the studies needle insertion was performed along a straight path. For a curved needle insertion, in which the path is almost circular, this 1-DOF translational model had to be mapped along a circular trajectory.

The suture movement is performed in the $y-z$ plane. Consequently, $\vec{F}_{y}$ and $\vec{F}_{z}$ pass through the needle insertion phases described in Figure 8(a). The force model along the $x$-axis does not vary because the needle insertion is always perpendicular to this axis (Figure 7). $\vec{F}_{x}$ simulates the reaction forces against the lateral needle displacement, as described in Kataoka et al. (2002). In real sutures, these forces help to keep the needle in place once it has been inserted (Figure $8(\mathrm{~b})$ ). Torque, $\tau_{x}$, is calculated by multi-

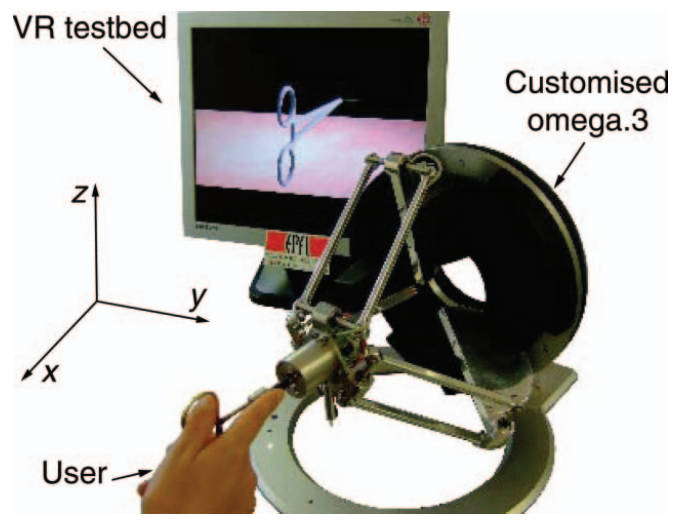

Figure 7. Customised Omega device used in the experiment and VR testbed.

plying the needle tip force, $\vec{F}_{r}$, which is tangential to the needle circle, by the needle radius.

With these definitions:

- $d\left(d_{x}, d_{y}, d_{z}\right)$ is the tissue deflection.

- $l_{1}$ is the thickness of the first layer, and $\left(d_{z}-l_{1}\right)$ represents the amount of needle penetration in the second layer with respect to the first.

- $K$ values are the elastic coefficients.

- $B_{1}$ and $B_{2}$ values are the damping coefficients per unit of length for each insertion phase.

- $B_{x}$ is the a damping coefficient along $x$-axis.

- $v\left(v_{x}, v_{y}, v_{z}\right)$ is the needle tip velocity.

Before puncture occurs $\left(d<d_{\text {threshold }}\right)$, the force model is described by

$$
\begin{aligned}
& \text { Shear component: } \vec{F}_{y}=K_{y 1} \cdot \vec{d}_{y}, \\
& \text { Normal component: } \vec{F}_{z}=K_{z 1} \cdot \vec{d}_{z} .
\end{aligned}
$$

After puncture $\left(d>d_{\text {threshold }}\right)$ :

Shear component:

$$
\vec{F}_{y}=K_{y 2} \cdot \vec{d}_{y}+\left(B_{y 1} \cdot l_{1}+B_{y 2} \cdot d_{y}\right) \cdot \vec{v}_{y},
$$

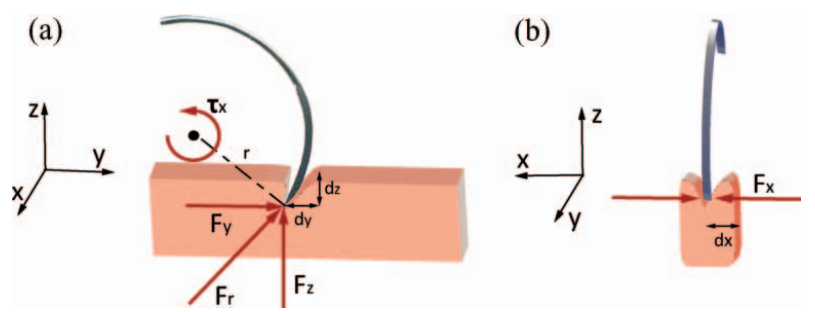

Figure 8. Interaction model description. (a) $F_{y}$ and $F_{z}$ components of the rendered force $F_{r}$ and torque $\tau_{x}$. (b) Force along the $x$-axis. 

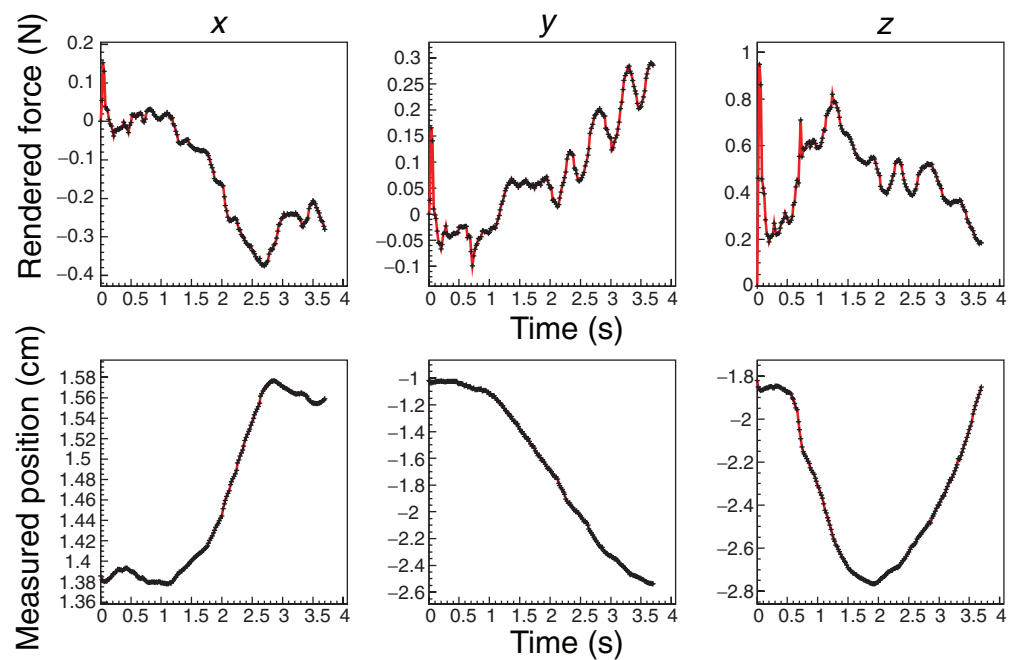

Figure 9. Interaction forces and needle tip position along each axis for a suturing trial. The initial peak of the force curve, especially along the $z$-axis, is due to the higher tissue stiffness before puncture.

Normal component:

$$
\vec{F}_{z}=K_{z 2} \cdot \vec{d}_{z}+\left(B_{z 1} \cdot l_{1}+B_{z 2} \cdot\left(d_{z}-l_{1}\right)\right) \cdot \vec{v}_{z} .
$$

Forces when extracting the needle:

$$
\text { Shear component: } \left.\vec{F}_{y}=\left(B_{y 1} \cdot l_{1}+B_{y 2} \cdot d_{y}\right)\right) \cdot \vec{v}_{y} \text {, }
$$

Normal component: $\vec{F}_{z}=\left(B_{z 1} \cdot l_{1}+B_{z 2} \cdot\left(d_{z}-l_{1}\right)\right) \cdot \vec{v}_{z}$.

The force model along the $x$-axis is constant:

$$
\vec{F}_{x}=K_{x} \cdot \vec{d}_{x}+B_{x} \cdot \vec{v}_{x} .
$$

Torque is calculated from the resulting force as

$$
\begin{aligned}
\vec{F}_{r} & =\vec{F}_{y}+\vec{F}_{z}, \\
\vec{\tau}_{x} & =\vec{r} \times \vec{F}_{r} .
\end{aligned}
$$

According to the visual simulation, when the needle tip deflects the tissue before the puncture occurs (before switching to the next needle node), the forces and the torque rendered to the user are determined by Equations (1), (3) and (9). In that phase, the $y$ and $z$ stiffness, $K_{y 1}$ and $K_{z 1}$, are higher, but suddenly decrease when the energetic threshold is overcome. Afterwards, the interaction model is mainly due to damping forces that depend on the amount of needle penetration into the tissue, as described by Equations (3) and (4). Therefore, the force model is non-linear with the $y$ and $z$ stiffness that are higher before the puncture and then decrease suddenly. In addition, when the user inverts the trajectory, which means that the needle is being extracted from the entrance point, the force model changes to the extracting phase.
The magnitudes of $K$ and $B$ were similar to the measurements of an ex-vivo liver puncture by Okamura et al. (2004) and the parameters used by Gerovichev et al. (2002). However, during the screening trials, subjects reported that the initial tissue stiffness felt was too high after puncture. When the spring force is released, there is an undesired overshoot in their movement. Subjects push against a boundary that is suddenly removed, thus they are not able to stop immediately due to both reaction time and arm impedance. The same phenomenon was identified by Gerovichev et al. (2002) and Barbé et al. (2007a) and is a factor that must be compensated for by the control scheme when performing teleoperated needle insertion. Therefore, initial stiffness was slightly reduced to minimise this effect.

In Figure 9, rendered forces and needle tip position in each axis during a needle insertion trial are shown. The peak force that appears at the beginning of the insertion, mainly along the $z$-axis, is due to the initial tissue stiffness before the puncture occurs.

This interaction model was tried and validated by surgeons, who affirmed that there was a good correlation between forces and the needle insertion and that it was realistic.

\subsection{Suture performance assessment}

There are many surgical procedures where sutures are key points, such as repairing liver lacerations, cardiac procedures etc. Also when suturing less delicate wounds, such as in dermatologic surgery, both cosmetic and functional results can be compromised if the execution is poor.

Kitagawa et al. (2005) investigated the sensory substitution effect in suture-manipulation forces for robotic surgical systems. In this study applied forces during a knot-tying 


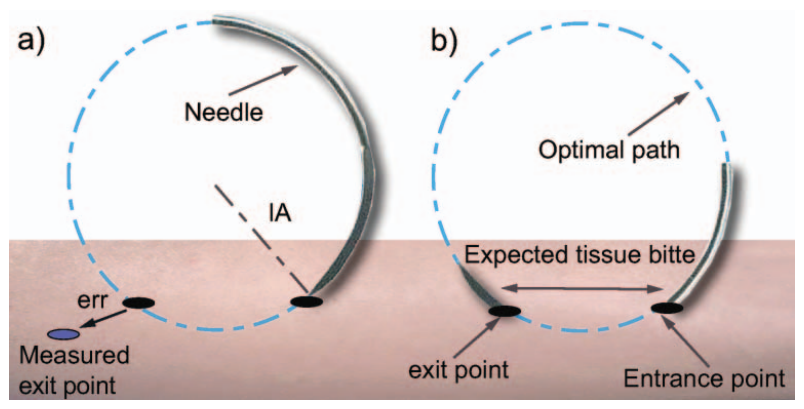

Figure 10. Optimal suture during curve-shape needle insertion described in Seki (1987), Iwamoto et al. (1993) and Solis et al. (2008).

task were measured in several sensory substitution scenarios. Results showed that when force feedback was given by visual or auditory modes, the applied forces approximate suture tensions achieved by manual suture. However, the trajectory of the needle during the insertion was not analysed.

Iwamoto et al. (1993) and Solis et al. (2008) defined the ideal suture as the one where the needle emerges exactly at the exit point with the intended tissue bite with the least possible suture-trauma (Figure 10(a)). In order to minimise trauma while placing a suture with its intended span and tissue bite in the expected place, the needle must advance along its curvature (Figure 10(b)). The position error highly depends on the insertion angle (IA) of the needle while entering the tissue. In addition, the choice of the optimal insertion angle is frequently related to the surgeon's experience (Seki 1987). According to these publications pinpoint accuracy in the suturing technique can be measured by the distance deviated from the optimal exit point. Therefore, in this experiment suture point error is calculated as the modulus of the $3 \mathrm{D}$ distance vector between the given suture point and the suture point reached by the subjects (err in Figure 10(a)). In addition to the position error in both exit and entrance suture points we measured maximum penetration depth into the tissue and task completion time, to check whether the feedback type affects these parameters.

\subsection{Experimental protocol}

Two different configurations of the experiment were performed. The second configuration took into account how experienced surgeons will normally perform the task. In the first configuration the subjects were sitting but the surgeons reported to perform an experiment were standing, so this factor was changed for the second configuration. In addition, in the first configuration the needle was grasped from the extremity (Figure 11(a)) instead of at one-third of its length as usually recommended (Figure 11(b)). Therefore, a second round of experiments was performed with subjects standing and the needle held correctly to study if there was any influence on the results.

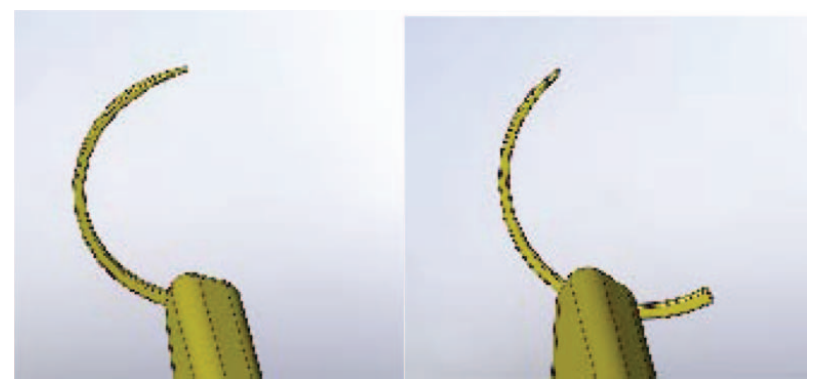

Figure 11. (a) Needle held at the extremity and (b) needle held at one-third of its length.

\subsubsection{Subjects}

The two configurations of the experiment were conducted with 10 subjects each, aged 25-35 years, all right-handed. The subject groups were composed of seven males and three females for the first configuration, and five males and five females for the second one. In both the configurations there were three subjects with suturing experience. These details are summarised in Table 2.

\subsubsection{Experiment}

During the experiments, three feedback conditions were used: (1) visual feedback only $\left(V_{F}\right),(2)$ visual and force feedback $\left(V_{F}+F_{F}\right)$ and (3) visual, force and torque feedback $\left(V_{F}+F_{F}+T_{F}\right)$. Two suture bite distances (14 mm and $12 \mathrm{~mm}$ ) were imposed to vary the sutures.

Before the experiments, users performed a training phase to familiarise themselves with the test: (1) the subject was asked to explore the tissue by changing the feedback condition during an unlimited time and (2) a training phase similar to the experiment was carried out. The aim of the training phase was to familiarise subjects with the task so that there would be no further learning effect during the experiment. The virtual testbed used in this experiment represents a section of visco-elastic tissue where the ideal suture entrance and exit points are indicated (Figure 3, right). Subjects are asked to introduce the curved needle as precisely as possible in the indicated location. The user starts by placing the needle on the reference entrance point and then performs the suture movement targeting the exit point (Figure 3 , left). A visual cue appears over the tissue to indicate that the suture is finished. The users are instructed to release

Table 2. Summary of the experimental characteristics for each configuration. Three out of 10 subjects had previous suturing experience.

\begin{tabular}{llll}
\hline $\begin{array}{l}\text { Experiment } \\
\text { configuration }\end{array}$ & $\begin{array}{c}\text { Subject's } \\
\text { posture }\end{array}$ & $\begin{array}{c}\text { Needle } \\
\text { position }\end{array}$ & $\begin{array}{c}\text { Subject's } \\
\text { gender }\end{array}$ \\
\hline First & Sitting & Fig. 11(a) & $7 \mathrm{M}, 3 \mathrm{~F}$ \\
Second & Standing & Fig. 11(b) & $5 \mathrm{M}, 5 \mathrm{~F}$ \\
\hline
\end{tabular}



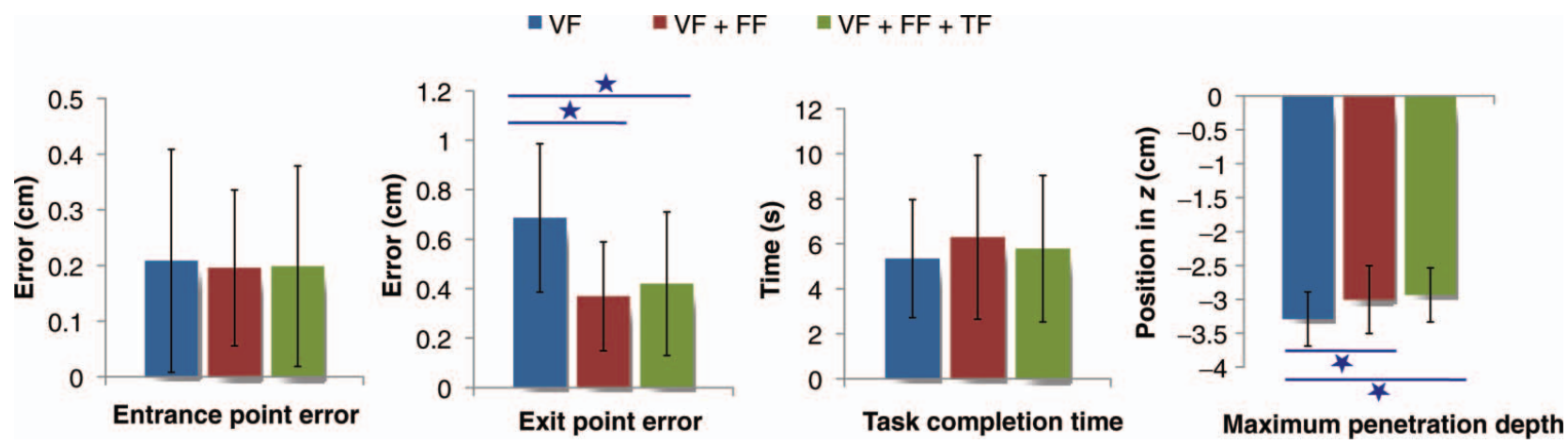

Figure 12. Mean values for each parameter with respect to the feedback condition (first configuration). Lines with stars above connect feedback conditions that have statistically significant different mean values for $\alpha_{B}<0.0167$.

the tissue and lift the tool towards this visual sign to make a pause. After three seconds the visual sign disappears and the next suture points are displayed. The distance between points, the location of the suture and the haptic conditions are changed in a random order. During the experiment, all combinations of distance between points and haptic conditions are repeated five times, thereby resulting in a total of 30 sutures per subject. Subjects are aware that time is measured; however, they are instructed to perform the suture as they would normally do, but trying to be as accurate as possible.

As mentioned in Section 2.4, the entrance and exit point errors, maximum penetration depth and task completion time are measured during the experiment. At the end of the experiment a questionnaire is given to the subjects. In this questionnaire the subjects' backgrounds, the task difficulty and their impressions of the different feedback conditions are requested.

\section{Results}

Given that the experiment follows a full factorial design with three factors (feedback condition, distance between suture points and individual subject), an Analysis of Variance (ANOVA) was performed to study their significance. The results of the ANOVA for the first experiment configuration are summarised in Table 3.

Table 3. ANOVA for the first experiment configuration: subjects were sitting and needle is taken from the extremity.

\begin{tabular}{lccc}
\hline Parameters & $\begin{array}{c}\text { Individual } \\
\text { subject }\end{array}$ & Distance & $\begin{array}{c}\text { Feedback } \\
\text { condition }\end{array}$ \\
\hline Exit point error & $P<0.001$ & $P>0.05$ & $P<0.001$ \\
$\begin{array}{l}\text { Entrance point error } \\
\text { Maximum penetration }\end{array}$ & $P<0.001$ & $P>0.05$ & $P>0.05$ \\
$\quad \begin{array}{l}\text { depth } \\
\text { Task completion time }\end{array}$ & $P<0.001$ & $P>0.05$ & $P<0.001$ \\
\hline
\end{tabular}

The individual subject factor has a significant effect on all parameters while the distance between suture points is not significant for any of them. These results mean that all parameters change significantly from one individual subject to another while distance between points does not affect any parameter. The feedback condition has a significant influence on the exit point error and the maximum penetration depth with a probability $P<0.001$, and for task completion time $(P<0.025)$.

The averaged values for each feedback condition are shown in Figure 12. The high standard deviation is due to the significance of the individual subject factor, as it is shown in the ANOVA results in Table 3. In order to assess whether the means of the three groups are statistically different from each other, a Bonferroni correction for multiplecomparison $t$-test was performed. Consequently, for an initial significance level, $\alpha=0.05$, the corrected significance level for the three comparisons is $\alpha_{B}=0.0167$. The Bonferroni $t$-test showed that $V_{F}+F_{F}$ and $V_{F}+F_{F}+T_{F}$ conditions significantly reduced the exit point error and the maximum penetration depth when compared with the $V_{F}$ condition. Nevertheless, the $V_{F}+F_{F}$ and $V_{F}+F_{F}+T_{F}$ conditions were not significantly different from each other. In addition, from the individual results shown in Figure 13, we observed that the exit point error presents the same tendency for all subjects with respect to the feedback condition. Note that the error with visual feedback only, $\left(V_{F}\right)$, is always higher than the feedback conditions, including force feedback $\left(V_{F}+F_{F}\right.$ and $\left.V_{F}+F_{F}+T_{F}\right)$. This proves that feedback conditions, including force feedback, significantly reduced the exit point error and the maximum penetration depth across all subjects independent of their individual tendency, although the addition of torque feedback to the haptic feedback does not imply any significant change.

Results of the ANOVA for the second configuration are shown in Table 4. As in the previous experiment, the subject factor has a significant influence on all the parameters while the distance between suture points is not significant for any of them. The feedback condition has a significant influence 

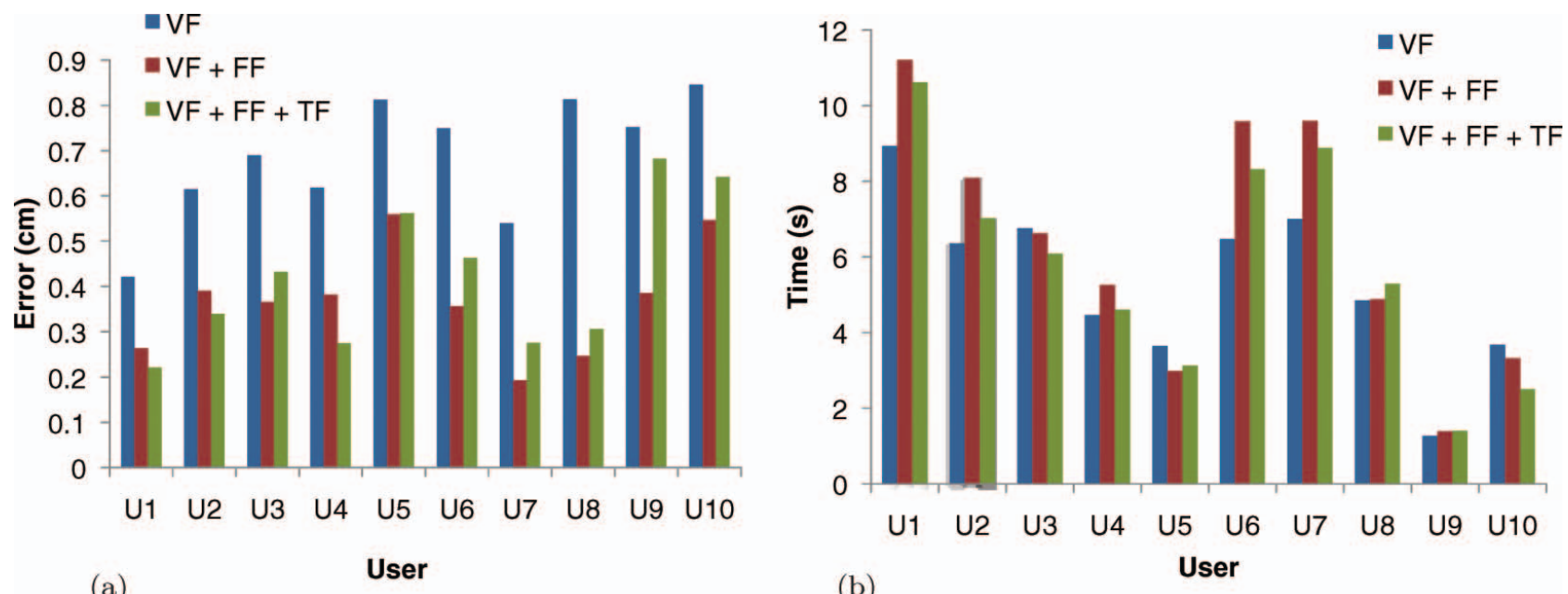

Figure 13. Results per subject and for each feedback condition (first configuration). U8, U9 and U10 correspond to subjects with suturing experience. Note that force feedback conditions highly reduce exit point error. (a) Exit point error per subject. (b) Task completion time per subject.

Table 4. ANOVA for the second experiment configuration: subjects were standing and held the needle at one-third of its length.

\begin{tabular}{lccc}
\hline Parameters & Subject & Distance & $\begin{array}{c}\text { Feedback } \\
\text { condition }\end{array}$ \\
\hline Exit point error & $P<0.001$ & $P>0.05$ & $P<0.001$ \\
$\begin{array}{l}\text { Entrance point error } \\
\text { Maximum penetration }\end{array}$ & $P<0.001$ & $P>0.05$ & $P>0.05$ \\
$\quad \begin{array}{c}\text { depth } \\
\text { Task completion time }\end{array}$ & $P<0.001$ & $P>0.05$ & $P<0.001$ \\
\hline
\end{tabular}

on the exit point error and maximum penetration depth with $P<0.001$ in both cases.

The mean values of each measurement for every feedback condition are shown in Figure 14. Once again a significant decrease of the exit point error and the maximum penetration depth is found for the two haptic feedback conditions when compared with only visual feedback $V_{F}$ for a sig- nificance level of $\alpha_{B}<0.0167$. No significant differences between the mean values of $V_{F}+F_{F}$ and $V_{F}+F_{F}+T_{F}$ conditions are observed.

We observed the same tendency for all subjects in Figure 15, with respect to the feedback condition, as it was found in the previous experiment. The feedback conditions, including force feedback $\left(V_{F}+F_{F}\right.$ and $\left.V_{F}+F_{F}+T_{F}\right)$ significantly reduce the exit point error and the maximum penetration depth as compared to visual feedback only $\left(V_{F}\right)$.

Overall, the results of the second configuration confirm the conclusions obtained in the first round. Only the entrance point error increased slightly mainly due to subject posture. In the first configuration, subjects were sitting and thus had more stability while manipulating the device. In both the configurations the entrance point error is only influenced by the individual subject factor. This is consistent as the location of the needle near the entrance point is totally based on visual cues. Accordingly, neither distance
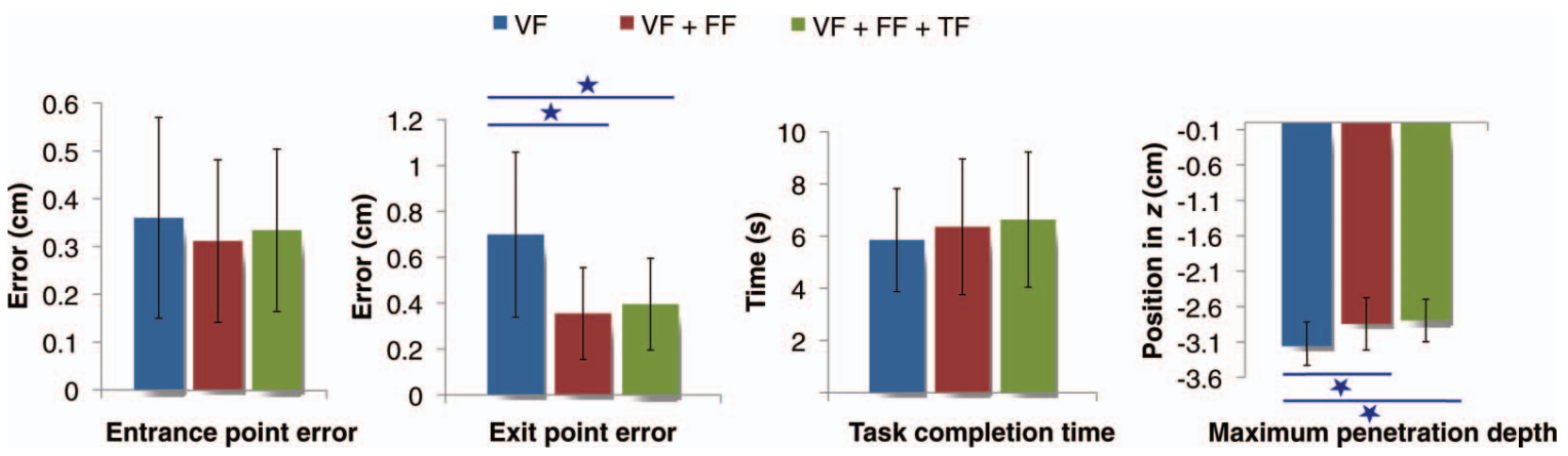

Figure 14. Mean values for each parameter with respect to the feedback condition (second configuration). Lines with stars above connect feedback conditions that have statistically significant different mean values for $\alpha_{B}<0.0167$. 


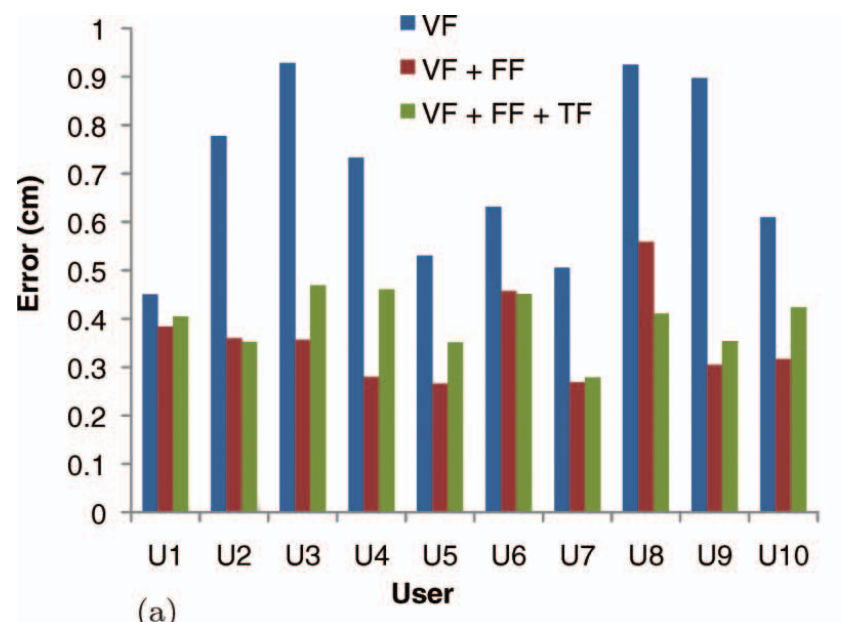

(a)

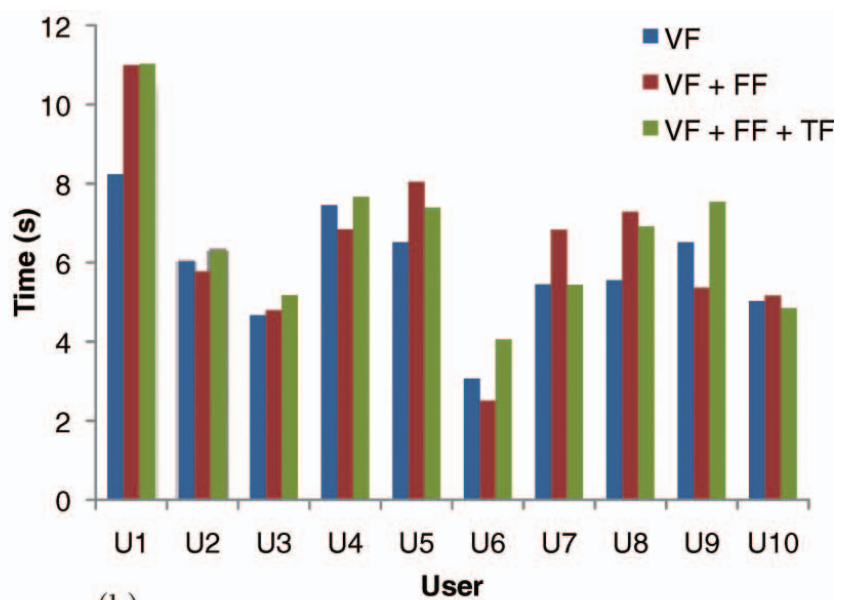

(b)

Figure 15. Results per subject and for each feedback condition (second configuration). U8, U9 and U10 correspond to subjects with suturing experience. Note that force feedback conditions highly reduce exit point error. (a) Exit point error per subject. (b) Task completion time per subject.

between suture points (bite) nor the type of feedback seem to have any effect on entrance point accuracy.

As can be seen in Figures 13(a) and 15(a), surgeons, results are quite similar to those of the untrained subjects. However, Figures 13(b) and 15(b) show that surgeons generally perform the sutures faster than other subjects.

In order to study the influence of user experience and experiment configuration, an ANOVA was again performed, merging data from both the experiments and adding two extra factors: user experience and experiment configuration. Results are shown in Table 5. Experiment configuration seems to have no significant influence on the results. User experience has a significant effect for the task completion time, which is lower for surgeons. However, the exit point error is similar for both surgeons and inexperienced subjects.

Normalised distributions of the results for the exit point error and the task completion time are shown in Figure 16. We observed that the surgeons' distribution for the task completion time is shifted to lower values compared to the unexperienced subjects' distribution. Nevertheless, their error distribution fits the same Gaussian as that of the unexperienced subjects. These results show that inexperienced subjects were specially concentrated in accuracy because they are not used to performing real sutures and ignore how fast this task is generally performed. This means that non-surgeons have a different subjective accuracy-speed tradeoff, which biases the results. Therefore, inexperienced subjects can perform sutures with similar accuracy to a surgeon but taking a significantly longer time. In addition, the effect of each subject's impedance on the overall performance should be taken into account. Several studies have shown that users can adapt their impedance to compensate the rendered forces by learning an internal model of the dynamics involved in that specific task (Shadmehr and Mussa-Ivaldi, 1994; Burdet et al. 2001). As surgeons have already performed the suture gesture and know the force profile of a needle insertion, they might have learned the optimal impedance that should be applied. Therefore, they can adapt their arms impedance to the force changes more quickly.

\subsection{Post-experiment questionnaire}

By analysing the answers of the questionnaire, it was found that the majority of the subjects found the task difficult in both the configurations, but that the second configuration caused more fatigue because they were standing during the entire experiment. All subjects noticed when the feedback condition was changed from one trial to another but more than half could not distinguish between

Table 5. Complete ANOVA results compared with user experience effects.

\begin{tabular}{lccccc}
\hline Parameters & Subject & Distance & Feedback condition & User experience & Experiment configuration \\
\hline Exit point error & $P<0.001$ & $P>0.05$ & $P<0.001$ & $P>0.05$ & $P>0.05$ \\
Entrance point error & $P<0.001$ & $P>0.05$ & $P>0.05$ & $P>0.05$ & $P>0.05$ \\
Penetration depth & $P<0.025$ & $P>0.05$ & $P<0.001$ & $P>0.05$ & $P>0.05$ \\
Task completion time & $P<0.001$ & $P>0.05$ & $P>0.05$ & $P<0.001$ & $P>0.05$ \\
\hline
\end{tabular}




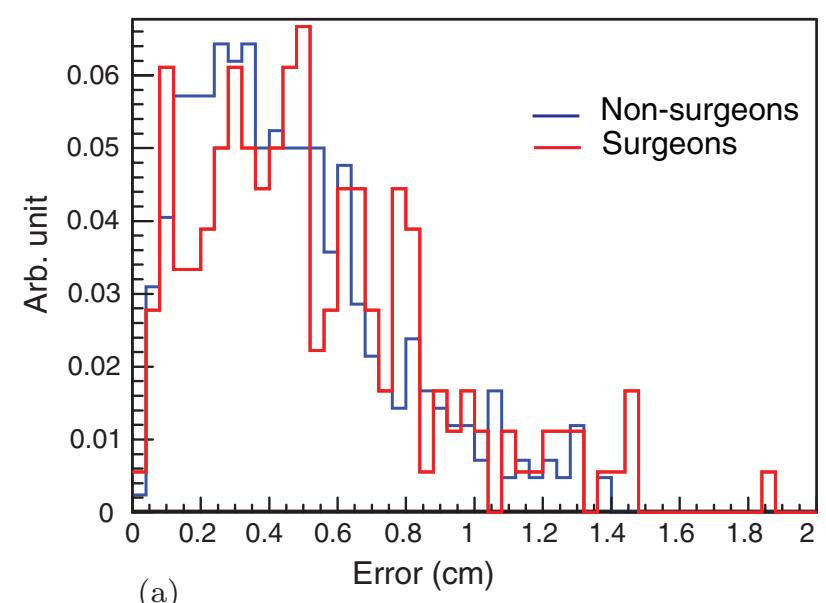

(a)

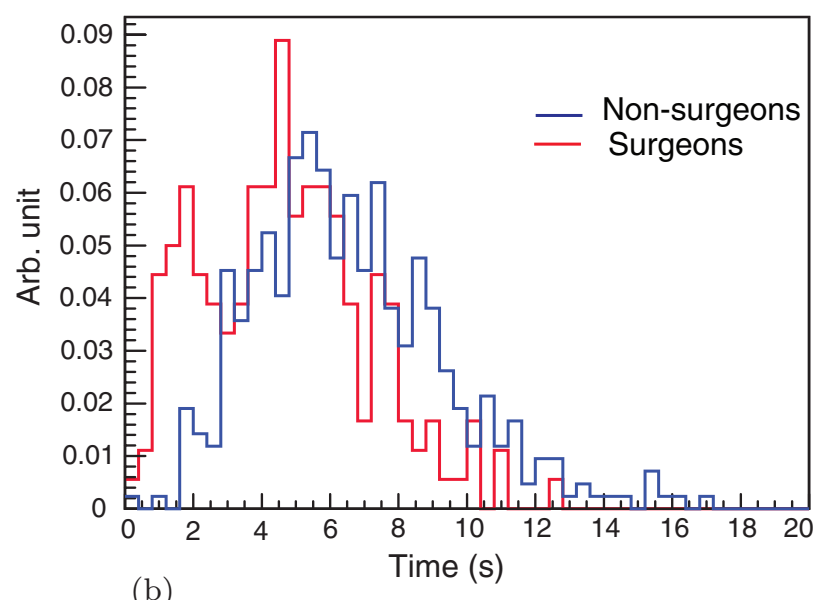

(b)

Figure 16. (a) Exit-point error distribution. (b) Task completion time distribution. A clear distinction can be seen between the surgeons' and non-surgeons' distributions for the task completion time.

force feedback alone and force plus torque feedback conditions. During the post-experiment questionnaire, most of the subjects asserted that they preferred the feedback conditions that included force feedback. It was felt more natural and the task was more intuitive than with only visual feedback.

\section{Discussion and conclusions}

This paper investigates the influence of force and torque feedback in a VR-based suturing task. We compare the deviation to the optimal suture points, task completion time and maximum penetration depth into the tissue in three feedback conditions: (1) visual feedback only, (2) visual and force feedback and (3) visual, force and torque feedback.

Based on this investigation, we can extract the following conclusions:

- Experimental results regarding the exit-point error confirm the assumption that force feedback can clearly ameliorate the accuracy in a suturing task.

- Without force feedback, users tend to penetrate too deeply into the tissue.

- The addition of torque feedback - in this particular context - does not improve suturing quality when compared to the addition of force feedback alone. This result supports the assumption that it might not be worth adding an extra actuator to provide torque feedback on the pronosupination DOF.

- These results are independent of the subject factor as all subjects present the same tendency with respect to the feedback condition.

- Force feedback significantly improves accuracy, for surgeons as well as non-surgeons. Generally, surgeons carried out the task considerably faster.
In real suturing tasks, the tissue helps to stabilise the needle trajectory by applying resistive forces against its movement. In addition, users exploit force information to correct their path and the applied force. Force feedback helps to stabilise the movement as tissue does in the real case. Consequently, force feedback clearly improves accuracy when compared to a free movement, which corresponds to the pure visual feedback condition in this experiment.

The majority of experienced subjects stated that they would be able to execute the task with only visual cues, as they do on current teleoperated systems such as da Vinci ${ }^{\mathrm{TM}}$ Surgical System (Guthart and Salisbury 2000). Nevertheless, their results showed that their error is almost halved when force feedback is provided, (e.g. user U8, U9 and U10 in both Figures 13 and 15). On the other hand, on a robotic surgery platform without force feedback, when the teleoperated robot inserts the needle, the real tissue stabilises it. However, contrary to a real suture, interaction forces are not transmitted to the surgeon, who might deviate, thereby applying undesired forces to the tissue. If these deviations are not cancelled out by the tremor filter, there will be significant risks of damaging fragile tissues. All results of this experiment clearly show that force feedback significantly improves accuracy independent of users' experience, and might thus speed up the surgeon's learning process. Torque feedback, in return, may not improve performance enough to justify the additional complexity and cost of torque feedback-enabled DOF.

Most of the subjects preferred the feedback conditions that included force feedback. These were perceived as being more natural and the task was more intuitive than with only visual feedback. Force feedback generally makes subjects feel more secure with the task as they have feedback to confirm their actions. This could also imply that the availability of force feedback might reduce the visual workload. 


\section{Acknowledgements}

We thank Force Dimension for their technical support. We also thank the surgeons of the visceral surgery department of the Geneva University Hospital (HUG) and the surgeons of the Lausanne University Hospital (CHUV) who willingly participated in the experiment. This work is funded by the European Commission in the framework of the ARAKNES FP7 European Project EU/IST- 2008-224565. Prof. Roger Gassert is supported by the NCCR Neural Plasticity and Repair, Swiss Science National Foundation.

\section{References}

Abolhassani N, Patel R, Moallem M. 2007. Needle insertion into soft tissue: a survey. Med Eng Phys. 29(4):413-431. Elsevier.

Barbagli F, Salisbury JK. 2003. The effect of sensor/actuator asymmetries in haptic interfaces. In: HAPTICS. IEEE Computer Society, p. 140.

Barbé L, Bayle B, De Mathelin M, Gangi A. 2007a. In vivo model estimation and haptic characterisation of needle insertions. Int J Robot Res. 26(11-12):1283-1301.

Barbé L, Bayle B, De Mathelin M, Gangi A. 2007b. Needle insertions modeling: identifiability and limitations. Biomed Signal Process Control. 2(3):191-198.

Bodner J, Wykypiel H, Wetscher G, Schmid T. 2004. EACTS; first experiences with the da vinci ${ }^{\mathrm{TM}}$ operating robot in thoracic surgery. Eur J Cardio-thoracic Surg. 25(5):844-851.

Burdet E, Osu R, Franklin D, Milner T, Kawato M. 2001. Nature publishing group; the central nervous system stabilises unstable dynamics by learning optimal impedance. Nature. 414(6862):446-449.

Cohen A, Chen E. 1999. Six degree-of-freedom haptic system as a desktop virtual prototyping interface. Asme Dyn Syst Control. 67:401-402. Citeseer.

Dasgupta P, Jones A, Gill I. 2005. Robotic urological surgery: a perspective. BJU Int. 95(1):20-23. Blackwell Science Ltd.

Ernst M, Banks M. 2002. Nature publishing group; humans integrate visual and haptic information in a statistically optimal fashion. Nature. 415(6870):429-433.

Gerovichev O, Marayong P, Okamura A. 2002. The effect of visual and haptic feedback on manual and teleoperated needle insertion. In: Medical Image Computing and Computer-Assisted Intervention, MICCAI 2002, Pt 1. Berlin: Springer Verlag.

Grange S, Conti F, Rouiller P, Helmer P, Baur C. 2001. Overview of the delta haptic device. In: Proceedings of Eurohaptics. Vol. 1. Citeseer, Birmingham, England: Harper Collins.

Guthart G, Salisbury J. 2000. The Intuitive TM telesurgery system: overview and application. In: IEEE International Conference on Robotics and Automation. Vol. 1. San Francisco, CA. USA. pp. 618-621.

Hayward V, Gregorio P, Astley O, Greenish S, Doyon M, Lessard L, McDougall J, Sinclair I, Boelen S, Chen X, et al.. 1998. Freedom-7: a high fidelity seven axis haptic device with application to surgical training. In: Experimental Robotics V. London: Springer Verlag, p. 443-456.

Hayward V, MacLean KE. 2007. Do it yourself haptics: part I. IEEE Robot Autom Mag. 14(4):88-104.

Iwamoto H, Osaki H, Sheki S. 1993. The technical skill in suturing: an analysis of the actual suture tracks. Surg Today. (23):800-806.

Jacobsen G, Berger R, Horgan S. 2003. Minimally Invasive Surgery Center, University of Illinois at Chicago Medical Center, USA. The role of robotic surgery in morbid obesity. J Laparoendosc Adv Surg Tech A. 13(4):279-283.
Kataoka H, Washio T, Chinzei K, Mizuhara K, Simone C, Okamura A. 2002. Measurement of the tip and friction force acting on a needle during penetration. In: Lecture Notes in Computer Science. London: Springer Verlag, p. 216-223.

Kitagawa M, Dokko D, Okamura A, Yuh D. 2005. Effect of sensory substitution on suture-manipulation forces for robotic surgical systems. J Thoracic and Cardiovas Surg. 129(1):151-158.

Lawrence D, Pao L, Salada M, Dougherty A. 1996. Quantitative experimental analysis of transparency and stability in haptic interfaces. Proc. ASME Dynamic Systems and Control Division, Atlanta, GA. DSC 58:441-449. Citeseer.

Lee J, Hwang I, Kim K, Choi S, Chung WK, Kim YS. 2009. Cooperative robotic assistant with drill-by-wire end-effector for spinal fusion surgery. Ind Robot-An Int J. 36(1):60-72. Emerald Group Publishing Limited.

Mack M. 2001. Am Med Assoc. Minimally invasive and robotic surgery. JAMA. 285(5):568.

Massie T, Salisbury J. 1994. The phantom haptic interface: a device for probing virtual objects. In: Proceedings of the ASME Winter Annual Meeting, Symposium on Haptic Interfaces for Virtual Environment and Teleoperator Systems. Vol. 55. p. 295 300.

McJunkin S, O’Malley M, Speich J. 2005. Transparency of a Phantom premium haptic interface for active and passive human interaction. In: Proceedings of the American Control Conference. Vol. 5. p. 3060.

Moser F, Horgan S. 2004. Robotically assisted bariatric surgery. Am J. Surg. 188(4S1):38-44. Elsevier.

Nio D, Bemelman W, Boer K, Dunker M, Gouma D, Gulik T. 2002. Springer; efficiency of manual versus robotical (Zeus) assisted laparoscopic surgery in the performance of standardised tasks. Surg Endoscopy. 16(3):412-415.

Okamura AM, Simone C, O'Leary M. 2004. Force modeling for needle insertion into soft tissue. IEEE Trans Biomed Eng. 51(10): 1707-1716.

Pheasant S. 1986. Bodyspace: anthropometry, ergonomics and design. London: Taylor Francis.

Samur E, Sedef M, Basdogan C, Avtan L, Duzgun O. 2007. A robotic indenter for minimally invasive measurement and characterisation of soft tissue response. Med Image Anal. 11(4):361-373. Elsevier Science.

Seibold U, Kuebler B, Hirzinger G. 2008. Prototypic force feedback instrument for minimally invasive robotic surgery. Medical robotics. Vienna, Austria: I-Tech Education and Publishing, p. 377-400.

Seki S. 1987. Accuracy of suture techniques of surgeons with different surgical experience. Japan J Surg. 17(6):465-469.

Seki S. 1988. Techniques for better suturing. Br J Surg. 75(12):1181-1184.

Semere W, Kitagawa M, Okamura AM. 2004. Teleoperation with sensor/actuator asymmetry: task performance with partial force feedback. In: HAPTICS. Washington, DC: IEEE Computer Society, p. 121-127.

Shadmehr R, Mussa-Ivaldi F. 1994. Adaptive representation of dynamics during learning of a motor task. J Neurosci. 14(5):3208. Soc Neuroscience.

Simone C, Okamura A. 2002. Modeling of needle insertion forces for robot-assisted percutaneous therapy In: Proceedings of IEEE International Conference on Robotics and Automation. Vol. 2. Citeseer, Washington DC: IEEE Computer Society, p. 2085-2091.

Solis J, Oshima N, Ishii H, Matsuoka N, Hatake K, Takanishi A. 2008. Towards understanding the suture/ligature skills during the training process using WKS-2RII. Int J Comput Assist Radiol Surg. 3(3):231-239. Springer. 
Toffin D, Reymond G, Kemeny A, Droulez J. 2007. Role of steering wheel feedback on driver performance: driving simulator and modeling analysis. Veh Syst Dyn. 45(4):375-388. Taylor $\&$ Francis.

Verner LN, Okamura AM. 2007. Effects of translational and gripping force feedback are decoupled in a 4-degree-of-freedom telemanipulator. In: WHC (World Haptics Conference). Washington, DC: IEEE Computer Society, p. 286-291.
Verner LN, Okamura AM. 2009. Force and Torque Feedback vs. Force Only Feedback. In: Third Joint Eurohaptics Conference and Symposium on Haptic Interfaces for Virtual Environment and Teleoperator Systems, Salt Lake City, UT, USA.

Wang F, Samur E, Burdet E, Bleuler H. 2008. Development of a microsurgery training system. In: Conf Proc IEEE Engineering in Medicine and Biology Society, 2008, p. $1935-1938$. 

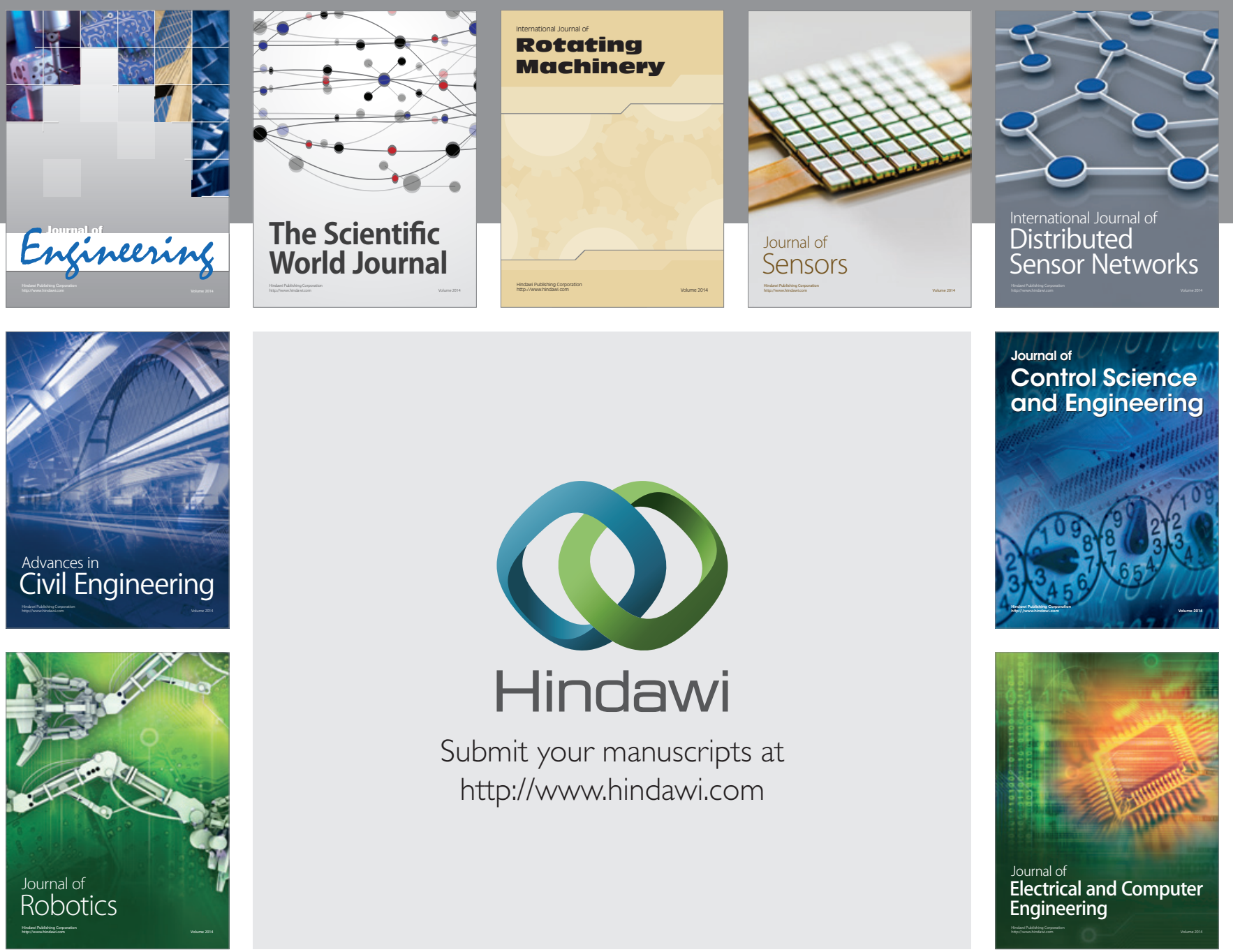

Submit your manuscripts at

http://www.hindawi.com
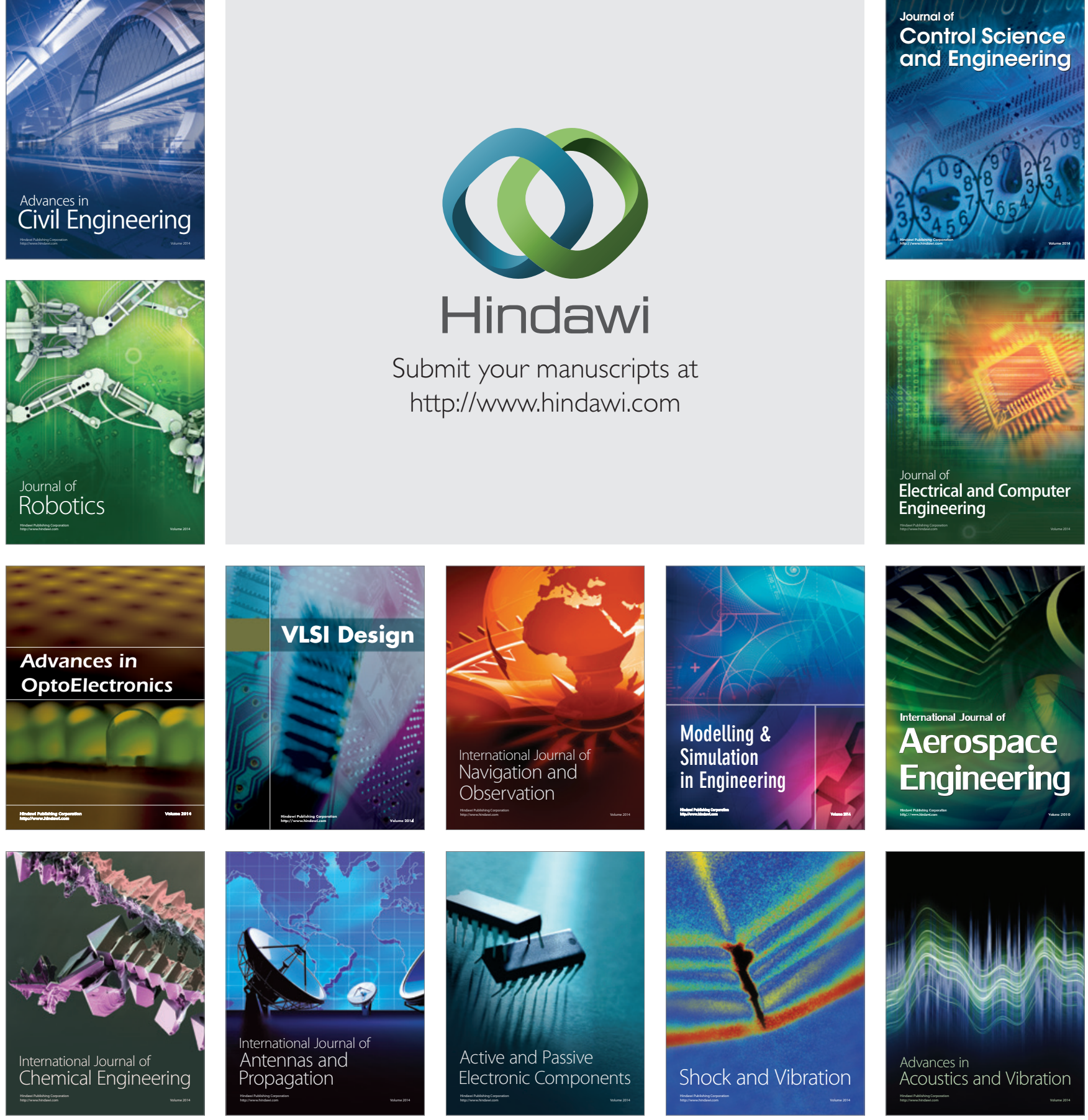